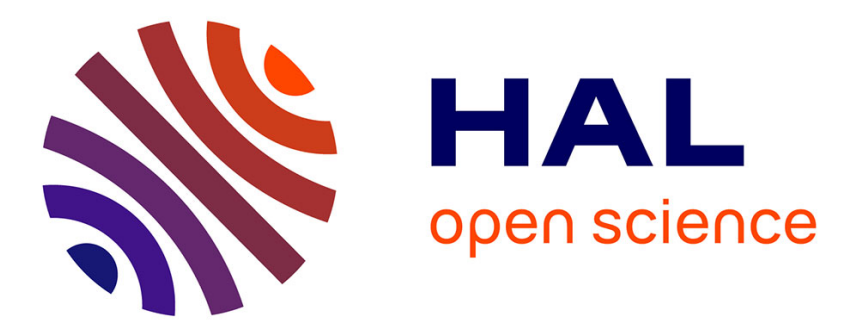

\title{
Étude de la variation au cours d'une période de la constante diélectrique complexe d'une cellule électroluminescente
}

\author{
D. Magnant, M. Durvy, H. Payen de La Garanderie
}

\section{To cite this version:}

D. Magnant, M. Durvy, H. Payen de La Garanderie. Étude de la variation au cours d'une période de la constante diélectrique complexe d'une cellule électroluminescente. Revue de Physique Appliquée, 1973, 8 (2), pp.101-103. 10.1051/rphysap:0197300802010100 . jpa-00243662

HAL Id: jpa-00243662

https://hal.science/jpa-00243662

Submitted on 1 Jan 1973

HAL is a multi-disciplinary open access archive for the deposit and dissemination of scientific research documents, whether they are published or not. The documents may come from teaching and research institutions in France or abroad, or from public or private research centers.
L'archive ouverte pluridisciplinaire HAL, est destinée au dépôt et à la diffusion de documents scientifiques de niveau recherche, publiés ou non, émanant des établissements d'enseignement et de recherche français ou étrangers, des laboratoires publics ou privés. 


\title{
REVUE DE PHYSIQUE APPLIQUÉE
}

\author{
Supplément au "Journal de Physique "
}

\section{Classification \\ Physics Abstracts \\ 18.40 \\ ÉTUDE DE LA VARIATION \\ AU COURS D’UNE PÉRIODE DE LA CONSTANTE DIÉLECTRIQUE COMPLEXE D'UNE CELLULE ÉLECTROLUMINESCENTE}

\author{
D. MAGNANT. M. DURVY et H. PAYEN DE LA GARANDERIE
}

Laboratoire de Luminescence, Faculté des Sciences de Poitiers, France

(Reçu le 4 avril 1972, révisé le 11 octobre 1972)

\begin{abstract}
Résumé. - Nous avons calculé, à partir de données expérimentales sur les courants traversant une cellule électroluminescente, les valeurs instantanées de la « constante » diélectrique complexe. Les variations de celle-ci sur une période ont pu être mises en relation avec celles de la luminance et de la puissance absorbée, permettant d'envisager un bilan énergétique de la cellule.

Abstract. - We have calculated from experimental data on electrical currents, in an electroluminescent cell, the values of the complex dielectric constant.

Its variations during a period are connected to brightness and absorbed power variations, permitting one to do an energy balance of the cell.
\end{abstract}

1. Dispositif expérimental. Méthode de calcul de grandeurs électriques. - La méthode du pont différentiel [1] appliquée à des cellules électroluminescentes $(\mathrm{ZnS}, \mathrm{Cu}, \mathrm{Cl})$ nous a permis d'obtenir sur un oscilloscope les courbes en fonction du temps, des courants total $I_{\mathrm{T}}$ et watté $I_{\mathrm{w}}$ qui traversent la cellule. Par construction nous obtenions également $I_{\mathrm{dw}}$ courant déwatté.

Par intégration (méthode des polynômes de Newton [2] appliquée à un ordinateur) il nous est possible de connaître la valeur $Q_{\mathrm{T}}$ de la quantité de courant en chaque point de la période.

Une cellule électroluminescente n'est pas équivalente à un condensateur parfait, ni exactement à une capacité parfaite en parallèle avec une résistance pure. En effet une analyse harmonique de $Q_{\mathrm{T}}$ nous montre que cette quantité n'est pas sinusoïdale, ni parfaitement en phase avec la tension.

$Q_{\mathrm{T}}$ peut s'écrire sous la forme :

$$
Q_{\mathrm{T}}=H_{\mathrm{OQ}}+\sum_{k=1}^{n}(A(\kappa) \cos \kappa \omega t+B(\kappa) \sin \kappa \omega t) \text {. }
$$

Expérimentalement, pour une tension de $250 \mathrm{~V}$ et une fréquence de $2500 \mathrm{~Hz}$, nous obtenons par l'analyse harmonique, les coefficients :

$$
\begin{array}{llll}
H_{\mathrm{OQ}}=0,01259 & & \\
A(1)=-0,01316 & B(1)=0,77180 & H_{1}=100 \% \\
A(3)=0,0058 & B(2)=0,00033 & H_{3}=0,08 \% \\
A(5)=0,0038 & B(3)=-0,00037 & H_{4}=0,06 \%
\end{array}
$$

$H_{\mathrm{OQ}}$ différent de zéro nous indique que le courant passe mieux dans un sens que dans l'autre, ce qui traduit l'effet redresseur de nos cellules électrolumi- nescentes. Ce résultat est en accord avec l'étude faite par Chukova et Fok sur des cellules symétriques du même type que les nôtres [3].

La relation $Q=C V(C=$ capacité de la cellule, $V=$ tension appliquée) ne peut pas être vérifiée directement puisque $Q$ n'est pas sinusoïdal alors que $V$ l'est. Il faut donc considérer $C$ sous la forme d'une quantité complexe liée à la constante diélectrique complexe $\varepsilon$ :

$$
\varepsilon=\varepsilon^{\prime}-j \varepsilon^{\prime \prime} \quad j=\sqrt{-1}
$$

et faire les calculs avec :

$$
v=V \mathrm{e}^{j \omega t} .
$$

De cette manière on pourra tenir compte, des éventuelles propriétés conductrices de la cellule électroluminescente.

2. Valeurs de la constante diélectrique complexe. - Pour pouvoir calculer les valeurs $\varepsilon^{\prime}$ et $\varepsilon^{\prime \prime}$ de la constante diélectrique complexe il faut exprimer ces valeurs en fonction des grandeurs électriques mesurées et calculées $I_{\mathrm{w}}$ et $Q_{\mathrm{T}}$.

La formule $Q=C V$ est remplacée par :

$$
Q=\frac{c_{0}}{\varepsilon_{0}} V\left(\varepsilon^{\prime}-j \varepsilon^{\prime \prime}\right) \mathrm{e}^{j \omega t}
$$

avec

$$
\varepsilon_{0}=\frac{1}{4 \pi \times 9 \times 10^{9}} \quad \text { et } \quad c_{0}=\frac{\varepsilon_{0} S}{e},
$$

$e$ étant la distance entre les armatures du condensateur et $S$ sa surface. 
La quantité $Q$ n'étant pas sinusoïdale nous admettrons que $\varepsilon^{\prime}$ et $\varepsilon^{\prime \prime}$ ne sont pas des constantes, mais sont des fonctions périodiques de $T=2 \pi / \omega$.

L'intensité totale du courant dans la cellule s'écrit :

$i=\frac{\mathrm{d} Q}{\mathrm{~d} t}=\frac{c_{0}}{\varepsilon_{0}}\left(\frac{\mathrm{d} \varepsilon^{\prime}}{\mathrm{d} t}-j \frac{\mathrm{d} \varepsilon^{\prime \prime}}{\mathrm{d} t}\right) V \mathrm{e}^{j \omega t}+$

$$
+\frac{c_{0}}{\varepsilon_{0}}\left(\varepsilon^{\prime}-j \varepsilon^{\prime \prime}\right)_{\mathrm{j}} j \omega V \mathrm{e}^{j \omega t}
$$

soit :

$$
i=\frac{c_{0}}{\varepsilon_{0}} v\left(\frac{\mathrm{d} \varepsilon^{\prime}}{\mathrm{d} t}+\omega \varepsilon^{\prime \prime}\right)+j \frac{c_{0}}{\varepsilon_{0}} v\left(\omega \varepsilon^{\prime}-\frac{\mathrm{d} \varepsilon^{\prime \prime}}{\mathrm{d} t}\right) .
$$

Nous pouvons donc écrire :

$$
\left\{\begin{array}{c}
i=i_{\mathrm{w}}+j i_{\mathrm{dw}} \\
i_{\mathrm{w}}=\frac{c_{0}}{\varepsilon_{0}}\left(\frac{\mathrm{d} \varepsilon^{\prime}}{\mathrm{d} t}+\omega \varepsilon^{\prime \prime}\right) v=\text { intensité wattée } \\
i_{\mathrm{dw}}=\frac{c_{0}}{\varepsilon_{0}}\left(\omega \varepsilon^{\prime}-\frac{\mathrm{d} \varepsilon^{\prime \prime}}{\mathrm{d} t}\right) v=\text { intensité déwattée }
\end{array}\right.
$$

Nous pouvons mesurer $I_{\mathrm{w}}$ par la méthode du pont différentiel. On peut écrire :

$$
i_{\mathrm{w}}=\frac{c_{0}}{\varepsilon_{0}} \omega \varepsilon^{\prime \prime} v
$$

en négligeant la quantité $\mathrm{d} \varepsilon^{\prime} / \mathrm{d} t$ devant $\omega \varepsilon^{\prime \prime}$. En effet avec les fréquences de la tension $v$ appliquée supérieures à $500 \mathrm{~Hz}$ que nous avons utilisées, $\omega \varepsilon^{\prime \prime}$ est grand. Par ailleurs l'étude expérimentale montre que les variations de $\varepsilon^{\prime}$ en fonction du temps sont très faibles.

Si on appelle

$$
Q_{\mathrm{dw}}=\frac{c_{0}}{\varepsilon_{0}} \varepsilon^{\prime} v
$$

laIpartie réelle de la quantité d'électricité, on obtient les expressions suivantes de $\varepsilon^{\prime}$ et $\varepsilon^{\prime \prime}$.

$$
\frac{c_{0}}{\varepsilon_{0}} \varepsilon^{\prime}=\frac{Q_{\mathrm{dw}}}{v} \quad \text { et } \quad \frac{c_{0}}{\varepsilon_{0}} \varepsilon^{\prime \prime}=\frac{i_{\mathrm{w}}}{v \omega} .
$$

Ces expressions nous permettent le calcul de $\varepsilon^{\prime}$ et $\varepsilon^{\prime \prime}$.

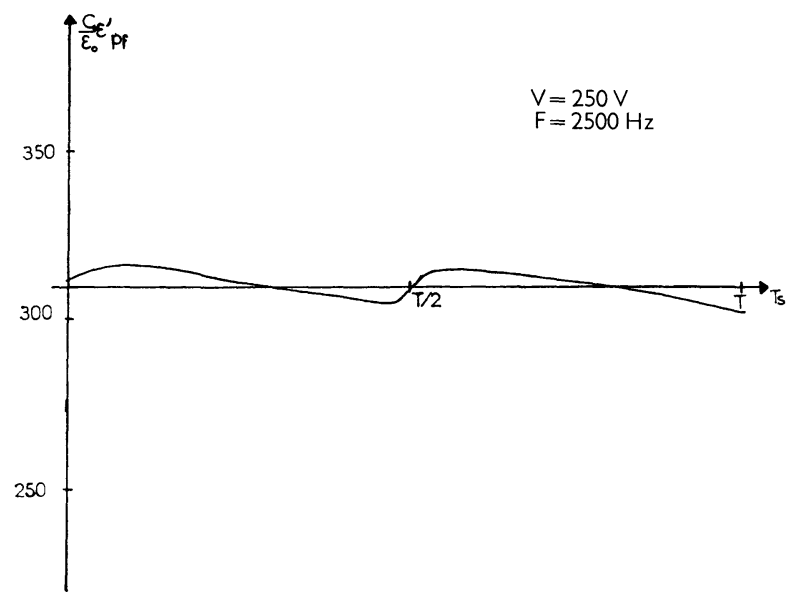

Fig. 1. - Evolution au cours d'une période de $\frac{c_{0}}{\varepsilon_{0}} \varepsilon^{\prime}$.

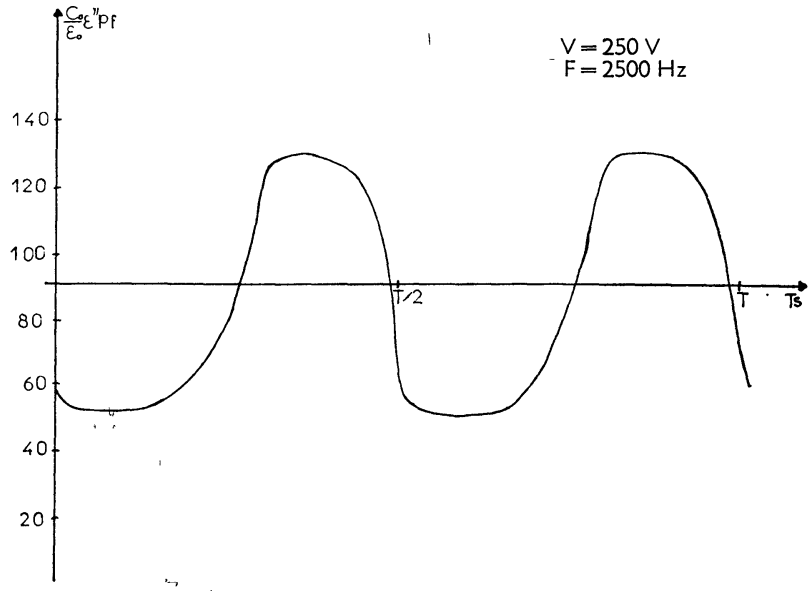

Fig. 2. -- Evolution au cours"d'une période de $\frac{c_{0}}{\varepsilon_{0}} \varepsilon^{n}$.

Nous donnons sur les figures 1 et 2 les variations en fonction du temps de $\varepsilon^{\prime}$ et $\varepsilon^{\prime \prime}$. Nous constatons que $\varepsilon^{\prime}$ peut être considéré comme constante sur la période alors que $\varepsilon^{\prime \prime}$ est une fonction périodique, de période double de celle de la tension appliquée.

Une cellule électroluminescente ne peut donc pas, du point de vue électrique être rendue équivalente à une capacité avec une résistance en parallèle.

3. Relation entre la constante diélectrique et la luminance émise. - Nous avons appliqué à $\varepsilon^{\prime \prime}$ la méthode d'analyse de Fourier que nous avions programmée dans un travail antérieur [4]. Ce qui nous a permis de comparer les harmoniques $H_{\mathrm{i} \varepsilon^{\prime \prime}}$ de $\varepsilon^{\prime \prime}$ et $H_{\mathrm{i} L}$ de la luminance $L$.

Pour une tension $V=250 \mathrm{~V}$ et une fréquence $F=2500 \mathrm{~Hz}$ on a :

$$
\begin{array}{llll}
H_{0 \varepsilon^{\prime \prime}}=1,58080 & & \\
A(1)=-0,00850 & B(1)=0,00000 & H_{1}=2,88 \% \\
A(2)=-0,00516 & B(2)=0,28967 & H_{2}=100 \% \\
A(3)=-0,00850 & B(3)=0,00000 & H_{3}=2,88 \% \\
A(4)=-0,01269 & B(4)=-0,01515 & H_{4}=6,90 \%
\end{array}
$$

Nous constatons que l'harmonique d'ordre 2 pour $\varepsilon^{\prime \prime}$ comme pour $L$, est prépondérante. Elle sera prise comme référence.

Nous séparons dans notre étude $H_{2 \varepsilon^{\prime \prime}}$ du terme constant $H_{0 \varepsilon^{\prime \prime}}$ qui traduit les pertes classiques dans les diélectriques imparfaits.

En module et en fonction de la tension nous avons :

$$
H_{2 \varepsilon^{\prime \prime}} \sim \exp \left(-\frac{\theta_{2}}{\sqrt{V}}\right) .
$$

Nous voyons (Fig. 3) que $\theta_{2}$ est voisin de $\gamma_{2}$ et $\gamma_{4}$ coefficients de l'exponentielle pour $H_{2 L}$ et $H_{4 L}$. Une étude en fréquence nous a montré également des variations semblables pour ces trois quantités. L'harmonique d'ordre 2 de $\varepsilon^{\prime \prime}$ vérifie donc une loi du type de celle trouvée par Destriau pour la luminance. 


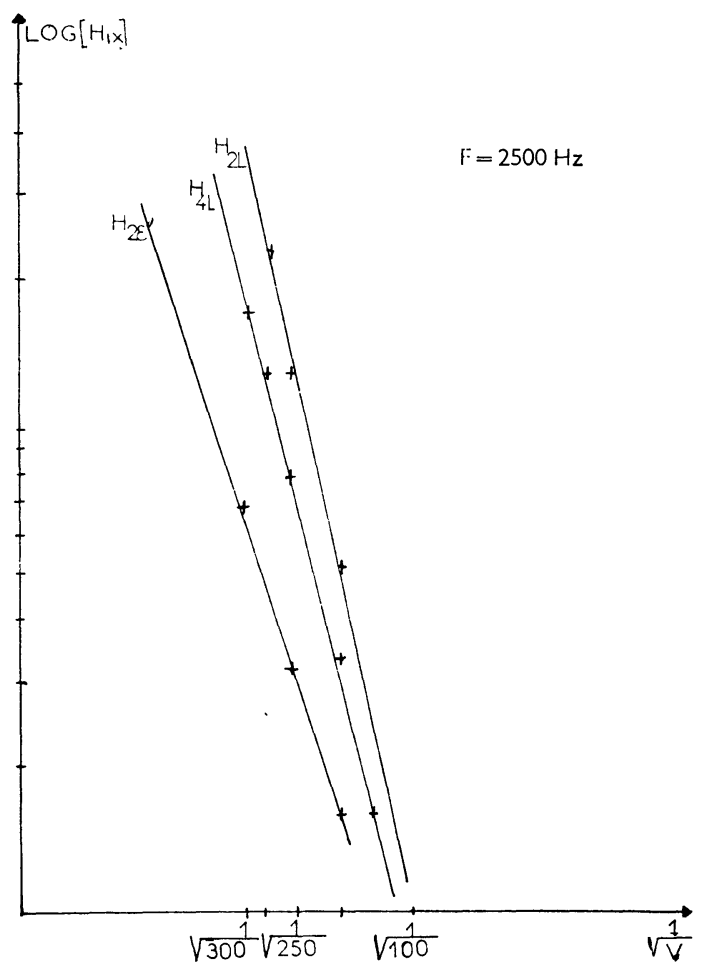

FIG. 3. $-\log \left(H_{\mathrm{ix}}\right)=f(1 / \sqrt{V})$.

D'autre part, si nous considérons le module de l'harmonique 2 de $\varepsilon^{\prime \prime}$,

$$
H_{2 \varepsilon^{\prime \prime}}=\sqrt{A(2)^{2}+B(2)^{2}}
$$

et que nous traçons $\log \left(H_{2 \varepsilon^{\prime \prime}}\right)$ en fonction de $\log (L / F)$ nous obtenons (Fig. 4) une variation identique à celle trouvée par Lehmann [5] en considérant la constante diélectrique sous la forme :

$$
\varepsilon_{2}^{\prime \prime}=\varepsilon_{20}^{\prime \prime}+\Delta \varepsilon_{2}^{\prime \prime}
$$

$$
\left\{\begin{aligned}
\varepsilon_{20}^{\prime \prime}= & \begin{array}{l}
\text { constante indépendante de l'électrolumi- } \\
\text { nescence }
\end{array} \\
\Delta \varepsilon_{2}^{\prime \prime}= & \begin{array}{l}
\text { partie variable en relation avec la lumi- } \\
\text { nance. }
\end{array}
\end{aligned}\right.
$$

L'analyse de Fourier nous permet de séparer directement les composantes de $\varepsilon^{\prime \prime}$ qui peuvent être liées avec l'électroluminescence.

Cette étude nous montre donc que la partie imaginaire de la constante diélectrique complexe n'est pas constante sur une période, mais variable en fonction du temps. D'autre part, nous pouvons dire que ces variations sont bien dues au phénomène d'électroluminescence, puisqu'on peut les relier à celle de la luminance.

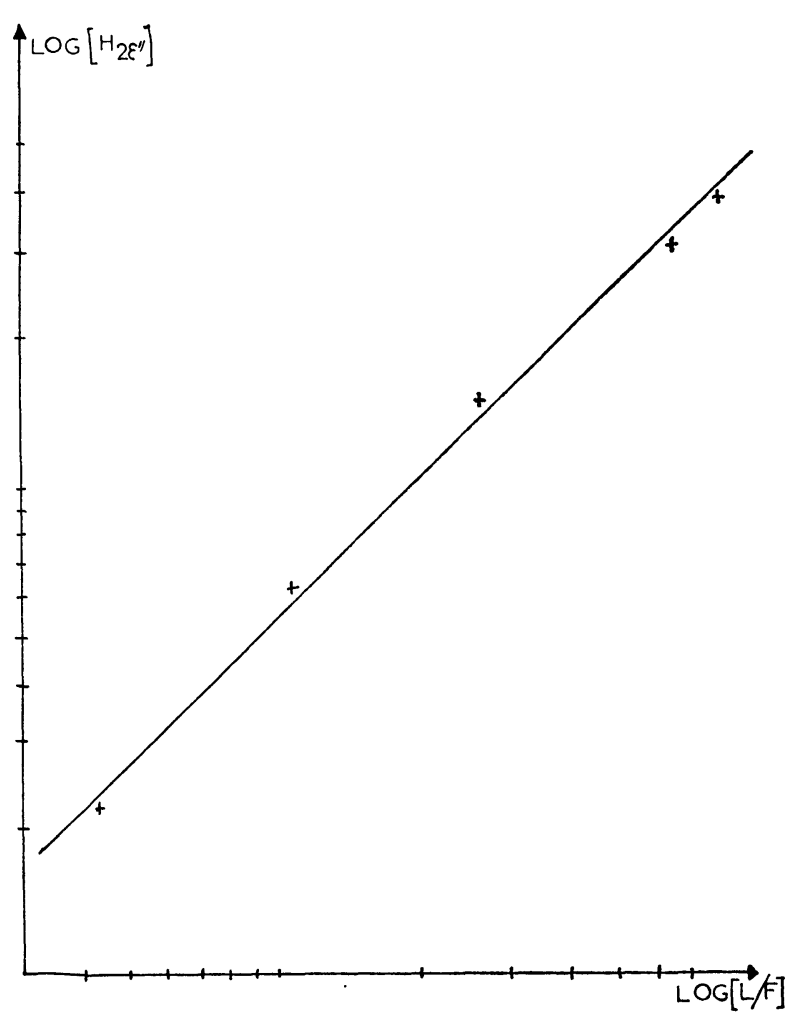

FIG. 4. $-\log \left(H_{2 \varepsilon^{\prime \prime}}\right)=f(\log L / f)$.

Ces variations peuvent trouver leur origine dans les modifications apportées dans la conduction par les mouvements des porteurs libres dans les phénomènes de piégeage et de recombinaison.

4. Conclusion. - Les relations trouvées entre la partie imaginaire $\varepsilon^{\prime \prime}$ de la constante diélectrique complexe et la luminance $L$ sont du même type que celles trouvées précédemment [4] pour la puissance wattée $P_{\mathrm{w}}$. Il semble bien que ces trois phénomènes soient étroitement liés.

Mais seule l'harmonique d'ordre 1 de $I_{\mathrm{w}}$, qui donne naissance à l'harmonique d'ordre 2 de $P_{w}$, conduit à une puissance moyenne consommée non nulle. Cependant, l'harmonique d'ordre 4 de la puissance wattée étant reliée d'une part à celle d'ordre 3 de l'intensité wattée, d'autre part à celles d'ordre 2 et 4 de la luminance, a bien un rapport avec l'énergie transformée en lumière. Pour expliquer la présence de cet harmonique nous avons pensé faire intervenir une force contre-électromotrice « $e$ » de même période que la tension, cette f. c. e.m. pouvant être calculée à partir de la portion de puissance wattée qui est transformée en lumière.

\section{Bibliographie}

[1] Georgobiani, A. N., L'Vova, E. Yu. and Fock, M. V., Opt. Spectrosc. 15 (1963) 142.

[2] Angot, A., Compléments de Mathématiques.

[3] Chukova, Yu. P. and Fok, M. V., Sov. Phys. Tech. Phys. 10 (1965) No 4.
[4] Magnant, D. et Payen de la Garanderie, H., Revue Phys. Appl. 6 (1971) 361.

[5] LehmanN, W., Dielectric behavior of electroluminescent zinc sulfide. J. Electrochemical Soc. 103 (1955) 24. 